\title{
Challenges to establish new medical technologies, that are not yet contractually stipulated with the insurers, into the daily medical routine
}

Type of article: Editorial

\author{
Wolfgang Seger \\ Chairperson of health science section at Medical Technologies Journal. \\ University of Bremen, Germany \\ Wolfgang.Seger@mdkn.de
}

\begin{abstract}
Advances in medical care are to a large extent brought forward by new medical technologies. Their implementation into daily medical routine requires a careful handling to be of rational use for individual patients and to avoid harm as well. For this responsible task critical key questions are listed for the deciding medical doctor treating an individual patient, for a conclusive scientific evaluation and as a decision support for insurers and policy makers to assess the economic potentials and regulatory challenges of new medical technologies and innovations.
\end{abstract}

Keywords: New Medical Technologies, Key Questions, Evidence Based Medicine, Health Care, Contracts.

\section{Introduction}

Progress in medical treatment is basically driven by the human genius to search for, invent, and recommend new medical technologies after examining pros and cons exhaustively. However, the decision to apply non-contractual medical technologies is often based on high hopes, core beliefs or principles and / or commercial interests. Therefore caution and a critical reluctance are advisable before the medical doctor is confident that his patients will actually benefit from the new approaches to the treatment. Scientific institutions should have found positively evaluated results based on the respective state of the art of medical findings and insurances should provide a comprehensive and nationwide financing for their insured persons contractually guaranteed.

\section{The following Key Questions are recommended for}

- the medical doctor treating an individual patient $(1,2)$ :

$>$ In case of an insured person: Is the designated treatment required by the contract or is it non-contractual?

$>$ Is there a therapeutic consequence for the individual when applying the new diagnostic method?

$>$ Is the relevant diagnosis certain and are the diagnostic prospects exhausted? If not, further diagnostic investigations are necessary. 
Are there no other methods contractually guaranteed to reach the aims of treatment with similar prospects of success?

$>$ Is there an adequate probability to induce a positive effect on the course of disease at the least?

$>$ Is the evaluation of risks of the new method compared to its advantages positively rated for the individual patient?

$>$ Which are the expected, desired or non desired outcomes applying the new method to this particular patient?

$>$ Is the illness life-threatening with an obviously reduced life expectancy so that the state of an emergency occurred to apply the new method as "ultima ratio"?

\section{- the scientific evaluation $(1,2)$ :}

$>$ Are the effectiveness and risks of the method referring to the indication proven by a sufficient number of cases and by applying scientifically correct statistics to allow a reliable evaluation?

$>$ Which is the level of studies according to evidence based medicine (EBM), when integrating clinical experience and patient values with the best available research information?

$>$ Which are the results categorizing different types of clinical evidence and ranking them according to the strength of their freedom from the various biases that beset medical research? Randomized and controlled studies and their systematic reviews are usually attributed to the highest value of perception. But one has to be careful when transferring the results to multimorbid elder people, because they are usually excluded from studies which usually concentrate only on one disease or one method.

$>$ Are there studies performed in several centres, independent from each other, confirming the results at the same or differing level of evidence?

$>$ Is the verification of methodical effectiveness severely impeded? This may be the case if diseases are seldom occurring and the etiology and course of the disease is not clear.

$>$ Is there a broad feedback following the discussion of experts? This may be documented by the number and quality of publications, publication in peerreviewed medical journals, meta-analyses, evaluation of reviews, textbooks, guidelines, expert opinions and consensus conferences and editorials. Multiple publications authored by the same study group with changing first authors do not account for a broad resonance specialist discussion.

$>$ Is the method applied by a substantial number of medical doctors? Epidemiologic health-care data are useful.

\section{- Insurers and Policy Makers to evaluate economic potentials and regulatory challenges $(1,2)$ :}

Which are the costs for an individual?

Which is the cost-benefit ratio for an individual?

$>$ Which is the cost-benefit ratio for the community of all insured persons?

$>$ Which is the cost-benefit ratio with regard to the long range impact assessment? 
Which is the cost-benefit ratio compared to other methods with equal outcome effects?

$>$ Which are the necessary regulations to initiate in case the new method should be transferred into routine health care?

$>$ Which are the necessary regulations to withdraw elder and no longer necessary methods from the market or in social health systems from a financing through the social health community?

$>$ Which are the necessary regulations to initiate and secure a sound financing and controlling of the insurances financing?

$>$ Which are the necessary regulations to sanction medical doctors applying not recognized, useless or even harmful new technological methods in the treatment of their patients?

An evaluation applying the same method in all cases may not be appropriate for any disease, any method of new medical technologies or any issue. It must be adjusted to the medical technology itself, the claimed indication and the relevant clinical parameters. Furthermore, the evaluation of obtained information that is relevant to solve the upcoming questions must be executed sophisticated.

The evaluation is respectively carried out considering the pursued aim of treatment on an individual basis or considering the community of all insured persons. A critical appraisal of relevant information is needed to find an answer to the above mentioned key questions referring to original research articles. Abstracts are not sufficient to appreciate the quality of a publication since they do not allow the evaluation of the evidence based integrity of the study and its results.

Poor evidence leads to poor clinical decisions. Following the above mentioned key questions may be helpful to make decisions about the individual and / or massive scale application of new medical technologies to the best medical knowledge and consciousness. Its goals are to secure the best care quality corresponding to the state of the art and to protect patients against commercially led, ideologically superimposed or personally guided interests. Orienting towards a sound scientific basis and respecting the framework conditions of the varying medical care systems may help to optimize the information, cooperation and informed consent of patients, medical doctors, insurers, health policy makers and lawyers.

\section{Declaration of conflicts}

No conflicts

\section{Authors' biography}

Prof. Dr. med. Wolfgang Seger, Professor for Rehabilitation Medicine, University of Bremen, Germany. Specialist in Internal Medicine, Gastroenterology, Social Medicine and Rehabilitation Medicine. Chairman of the Medical Health Advisory Board of the Statutory German Federal Association for Rehabilitation. Longstanding Medical Director and Deputy 
Medical Technologies Journal, Volume: 1, Issue: 4, October-December 2017, Pages:76-136. Doi :

https://doi.org/10.26415/2572-004X-vol1iss4

CEO, Advisory Board of all Statutory Health and Long Term Care Insurances in Lower Saxony, Germany, now freelance expert in Social Medicine.

\section{References}

1. Instructions manual to evaluate non-contractual new medical technologies (in German language): Begutachtungsanleitung Außervertragliche „Neue Untersuchungs- und Behandlungsmethoden (NUB)“, Medizinischer Dienst des Spitzenverbandes Bund der Krankenkassen e.V.(MDS) Essen, Germany, 2008, http://www.mds-ev.org

2. Cochrane Library with methods studies following evidence based medicine (EBM): http://www.cochranelibrary.com 